\section{Military Technical College Kobry El-Kobbah, Cairo, Egypt}

\section{$6^{\text {th }}$ International Conference on Electrical Engineering ICEENG 2008}

\title{
Novel design of a fractal monopole antenna for wireless communications
}

By

Hocine Kimouche*

M. Bitchikh*

B. Atrouz *

\section{$\underline{\text { Abstract: }}$}

In this paper, a new design of planar-tree fractal antenna based on ternary fractal tree geometry for multiband communication services is proposed, designed, and tested. The modified tree fractal antenna is employed to achieve multi-band characteristics. As a low-profile antenna, the proposed antenna covers the DVB-H (174-862 MHz), Bluetooth (2400-2484 MHz), WIMAX (Worldwide Interoperability for Microwave Access) and WLAN (Wireless Local Area Network) (5150-5356 MHz) band with $10 \mathrm{~dB}$ return loss criterion. The measured return loss of the proposed antenna is presented and compared with simulated results.

\section{Keywords:}

Multi-band Antennas, Fractal-Tree Antenna, Wireless Communications.

* Microwaves Laboratory, Polytechnic Military School, Bordj El Bahri, Algeria. E.Mail : hocine.kimouche@gmail.com 


\section{Introduction:}

There has been a strong trend in merging communications and computers using wireless means. This trend has been motivated by many applications, which require computing devices to be mobile, versatile and connected at all times. The wireless standards encompass many of the wireless computing uses for mobile communication.

The main application is a consumer market. However, other uses concern educational, industrial, and medical applications. For example, in schools or universities, there is a great need of accessing different resources (library, lectures or tutorials) which would be handy to accomplish using wireless means. In a hospital, wireless checking can allow patients to move around if adequate monitoring equipment exists. At an industrial workplace, monitoring can involve tracking of individual object needed for assembly while avoiding the clutter of wires. Therefore, wideband and multiband antennas designs have become increasingly important for mobile applications.

Nowadays, wireless antenna designers face many tough challenges. Not only do they have to fit multi-band, multi-purpose antenna systems into small stylish housings that are enticing to consumers, they also have to comply with an increasing number of regulation requirements, operator and end-user demands for radiation safety, highperformance functionality and quality.

The classics wire and patch antennas are intrinsically a narrow band devices. Their behavior is strongly dependent on the report of the antenna size to the working wavelength. The antennas parameters systems (gain, matching, and radiation pattern) endure then, any working frequency disagreement. One promising approach in this regards is to use fractal geometries to find the best distribution of currents within a given volume in order to meet a particular design goal.

Fractal geometries have been recently introduced in antennas design. It has been shown that fractal shaped exhibit characteristics that are associated with the geometric properties of fractal [1] [2]. One property associated with fractal geometry and used in antenna's design is self-similarity. A fractal antenna can be designed to receive and transmit over a wide range of frequencies using self similar properties associated with fractal geometry structures, because different antenna's parts are similar to each other at different scales. The second property is the efficiency of space filling of some fractal shapes which gives hope to reduce the antennas size, comparatively to that of classic antennas.

Various fractal shapes that possess self-similarity have been applied to multiband or miniaturized antenna design. A promising fractal geometry that ensures a successful design of a multiband antenna is known as the deterministic tree fractal. Multiresonant behaviors of the first-iteration tree mounted over a large conducting-ground plane are described in many papers [3] [4] [5]. However the conventional fractal-tree monopole antenna doesn't present many resonant frequencies in the band [0.2-6] $\mathrm{GHz}$ wireless 
band. Further, the poor matching property of the resonance frequencies has been shown. In this paper, a novel design of a miniature tree-fractal antenna is proposed.

\section{Antenna design:}

Fractal geometries are generated in an iterative fashion, leading to self-similar structures. The Tree geometry starts with a stem and allows one of its ends to branch off in two directions. In the next stage of iteration, each of these branches is allowed to branch off again. The process is continued endlessly as shown in figure (1):

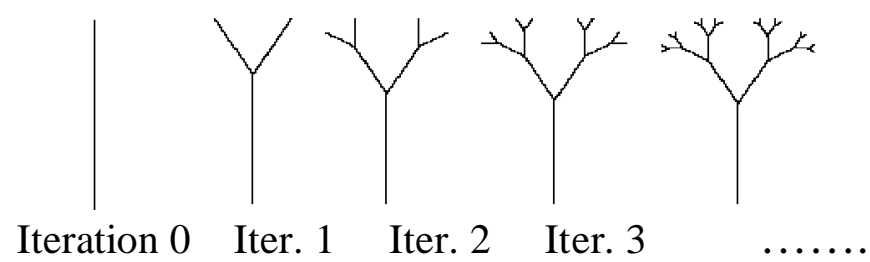

Figure (1): Fractal-tree geometry

It is possible to vary the scale factor between the length of the stem and that of branches. The procedure is known as the iterated function system (IFS) and is described by the matrix equation expressed as follows [6]:

$$
W_{1}\left(\begin{array}{l}
x \\
y
\end{array}\right)=\left[\begin{array}{ll}
\frac{1}{s} \cos \theta & -\frac{1}{s} \sin \theta \\
\frac{1}{s} \sin \theta & \frac{1}{s} \cos \theta
\end{array}\right]\left(\begin{array}{l}
x \\
y
\end{array}\right)+\left(\begin{array}{l}
0 \\
1
\end{array}\right)
$$

The new self-affine fractal geometry considered in this paper is constructed by using a deterministic ternary tree whose center is surrounded by a hexagonal shape as shown in figure (2). This is a first iteration. Then, the process is repeated at each branch center. This particular ternary-tree structure is closely related to the Sierpinski gasket. In fact this geometry can be interpreted as a wire-equivalent model of iteration 1 of Sierpinski gasket.

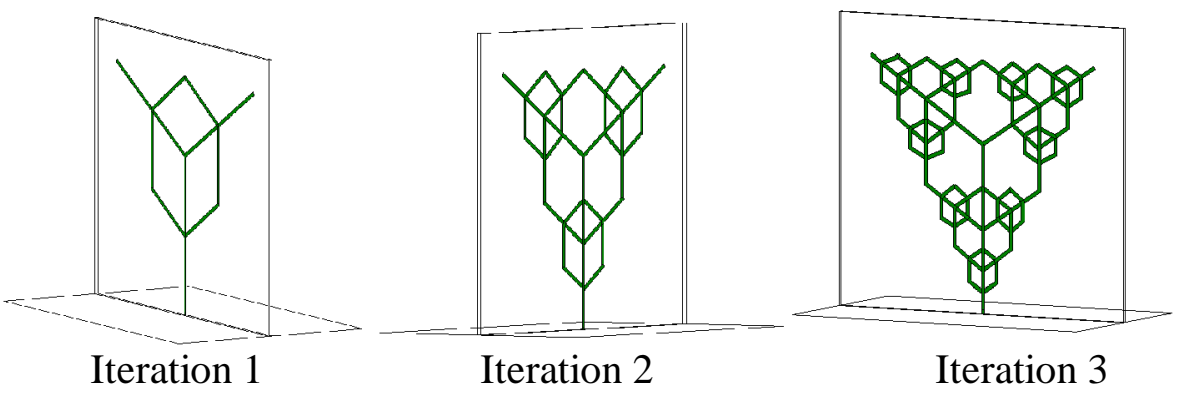

Figure (2): New fractal-tree monopole antenna over ground plane. 


\section{Fractal antenna simulation:}

Because of their geometric complexity, it is very difficult to predict mathematically the fractal antennas radiation pattern properties. The wide availability of powerful electromagnetic simulators makes possible the simulation of such problems, otherwise impossible to be solved.

Many numerical methods are available that predict the performances of such antennas. All these techniques are based on the resolution of the discrete form of Maxwell's equations. The most often used are the method of moments (MoM) and the Finite Difference Time Domain (FDTD) method.

The simulation software that has been adopted to investigate the properties of fractal antennas is NEC (Numerical Electromagnetic Code) based on the moment method. This software solves for the surface currents generated on perfectly conducting surfaces or thin wires, or the combination of both. From this current, the far field patterns and input impedances can be determined. Initial geometry estimation is obtained using (2) to determine the antenna resonance frequencies :

$h=\frac{\lambda_{0}}{4 \sqrt{\varepsilon_{e f f}}}$

With [7] :

$\varepsilon_{\text {eff }}=\frac{\varepsilon_{r}+1}{2}+\frac{\varepsilon_{r}-1}{2}\left[\left(1+12 \frac{h_{0}}{w}\right)^{-\frac{1}{2}}+0.04\left(1-\frac{w}{h_{0}}\right)^{2}\right]$

The antenna was designed to cover DVB-H (174-862 MHz), Bluetooth (2400-2484 MHz), WIMAX and WLAN (5150-5356 MHz). The position of the frequency bands may be controlled by proper adjustment of the scale factor or by the variation on the branching half angle. In our case, the branching half angle is fixed at $\theta=60^{\circ}$ and the scale factor is equal to $s=2$. The antennas are considered to have infinite ground plane. According to (2), the antenna height will be $h=48 \mathrm{~mm}$.

Three iterations were examined; figures (3) show the return loss. For easy comparison of performance, the return loss of basic tree is also included in the figures.

We immediately note that an additional resonant frequency appears in the band [0$6 \mathrm{GHz}]$. In addition, we note that the corresponding resonance is achieved with an excellent return loss of $-30 \mathrm{~dB}$ for the third iteration. The antenna is matched to $50 \Omega$ $\left(S_{11} \leq-6 \mathrm{~dB}\right)$ in the frequency ranges needed. Therefore, the required bands have almost been covered. 


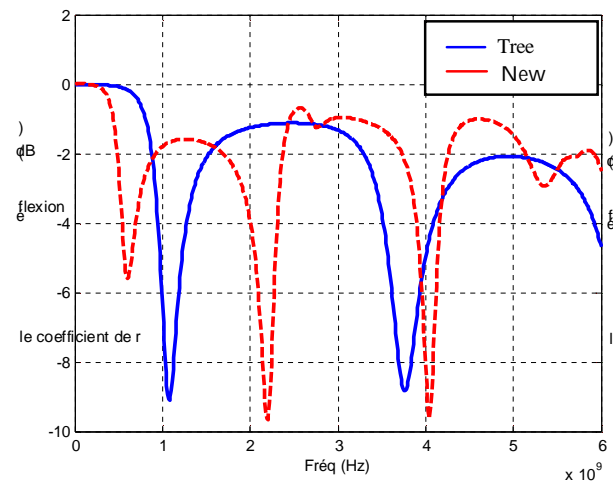

Iteration 1

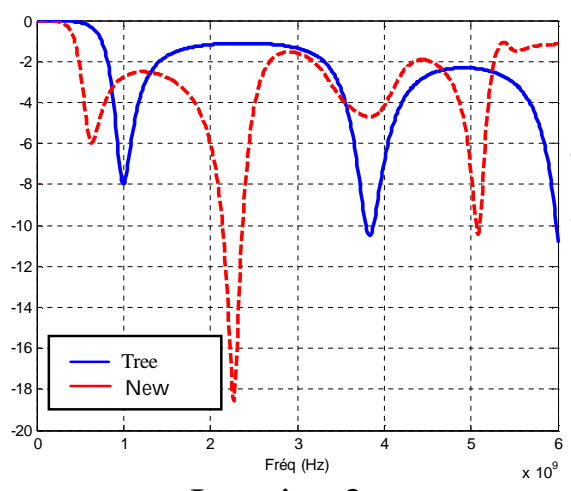

Iteration 2

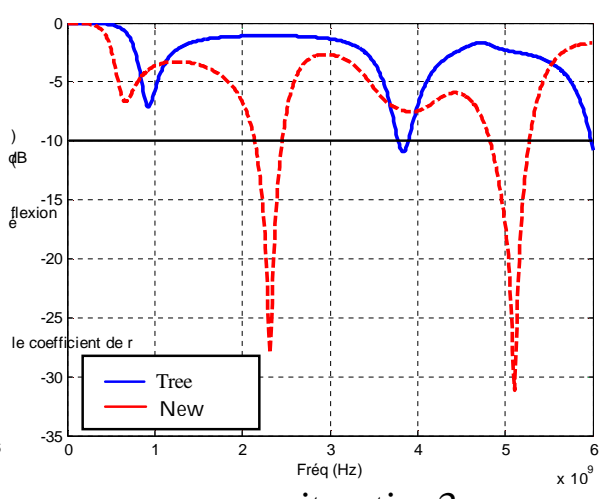

iteration3

Figures (3): Return loss for iteration 1, 2 and 3

We also record the antenna's radiation pattern for the three resonant frequencies (for iteration 3). The radiation characteristics are omnidirectional and seem to be roughly constant in the operating-frequency band.

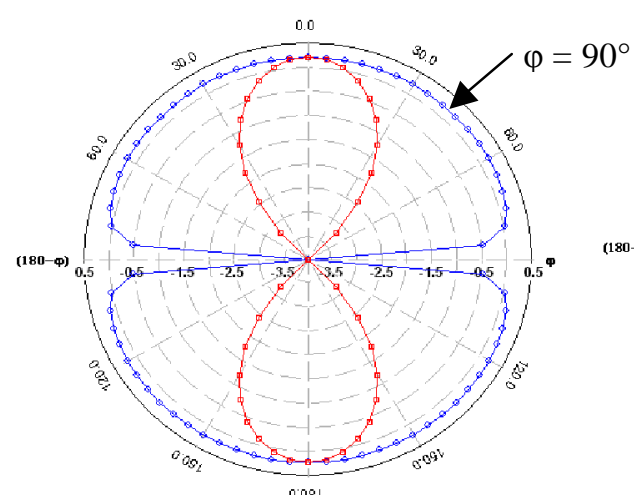

$\mathrm{f}=680 \mathrm{MHz}$

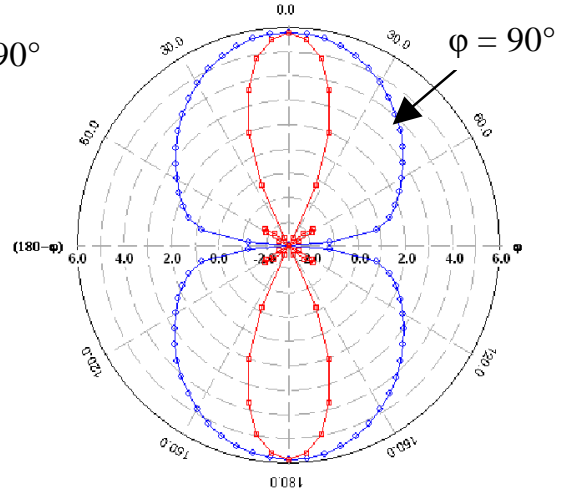

$\mathrm{f}=2330 \mathrm{MHz}$

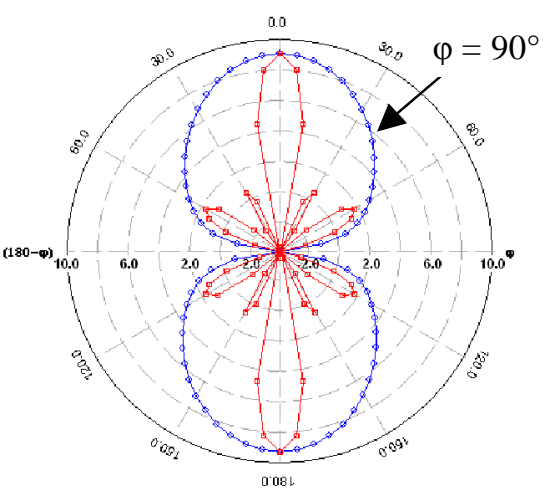

$\mathrm{f}=5100 \mathrm{MHz}$

Figures (4): Radiation pattern. $E_{\text {total }}\left(\right.$ for $\varphi=0^{\circ}$ and $\left.\varphi=90^{\circ}\right)$

Figure (5) shows the antenna gain versus frequency corresponding to the iteration 3. The antenna presents about $8 \mathrm{dBi}$ gain at $5100 \mathrm{MHz}$. 


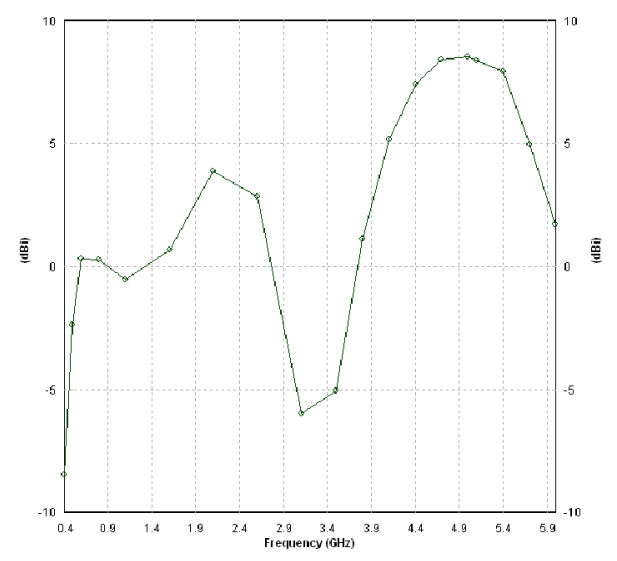

Figure (5): Antenna gain for iteration 3

\section{Experimental results and discussion:}

Three iteration with branching half angle of $\theta=60^{\circ}$ and height $h=4.8 \mathrm{~cm}$ were built and tested. Figure (6) shows the geometry of the novel antenna printed on a dielectric substrate and mounted over a $10 \times 10 \mathrm{~cm}^{2}$ conductor ground-plane. The antenna has been fed using a $50 \Omega$ coaxial probe by one stem. In this study, the dielectric substrate is FR-4 with thickness $h^{\prime}=1.6 \mathrm{~mm}$ and relative permittivity $\varepsilon_{r}=4.32$

The return loss is measured with an Agilent 8719ES network analyzer from $100 \mathrm{MHz}$ to $6 \mathrm{GHz}$.

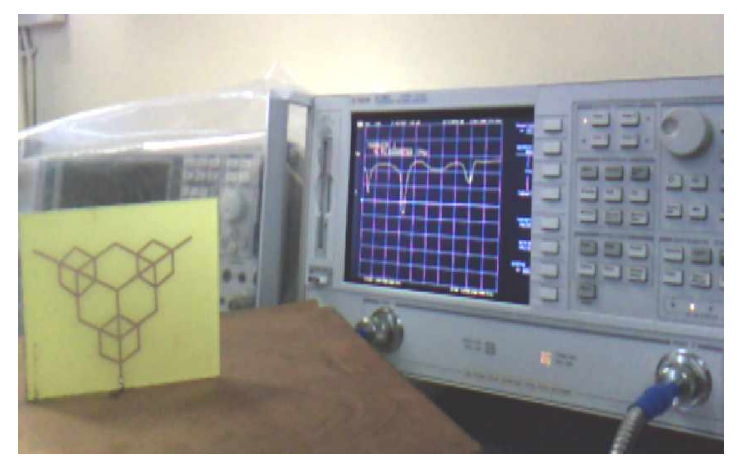

Figure (6): Antenna measurement with network analyzer

The return loss measurement for the first iteration is presented in figure (7). This curve confirms that the antenna has a multi-band character and has resonant frequencies located at required.

For the other iteration, the same behavior was noticed. The multi-band behavior is confirmed by the measure and an acceptable agreement between measurement and simulation is observed. 

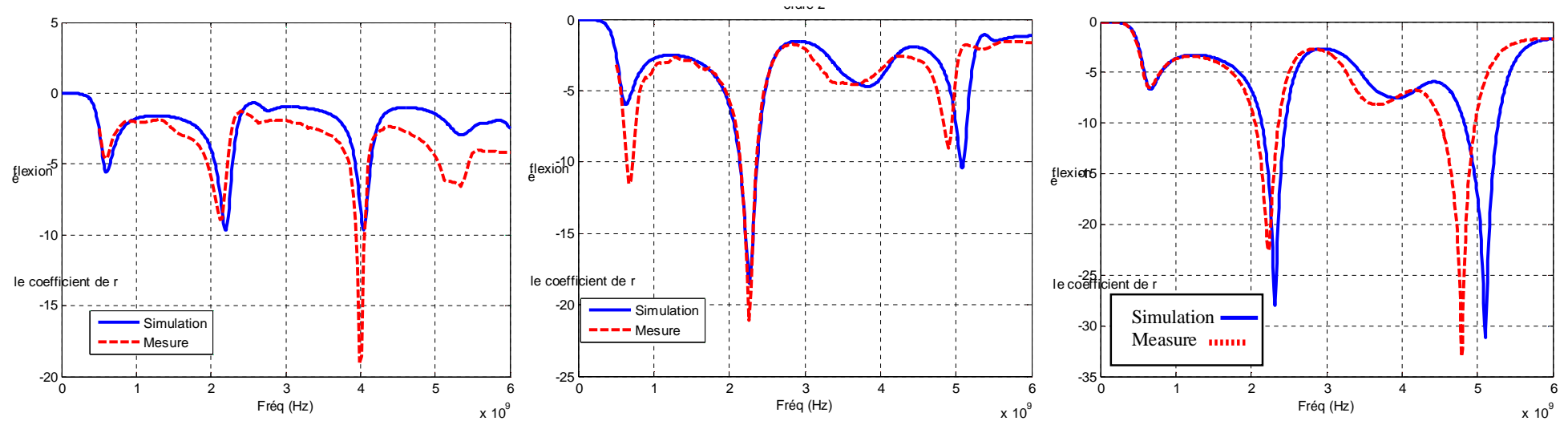

Figures (7): Return loss for iteration 3

\section{Conclusion:}

Both experimental and numerical results on the novel design of fractal-tree antenna have been presented. All of them describe multi-band behavior of the miniaturized antenna. This behavior is consistent from viewpoint of the input return loss and radiation patterns. A good agreement between simulation and measurement is obtained. The proposed use of printed circuit fractal antennas can be a part of telecommunications systems that have seen a revival lately due to numerous wireless applications.

\section{References:}

[1] D.H. Werner, S. Ganguly, An overview of fractal antenna engineering research', IEEE. Antennas and Propagation Magazine. Vol. 45, n¹, Feb. 2003.

[2] Cohen, N, Fractal Antenna Applications in Wireless Telecommunications, Professional Program Proc. of Electronics Industry Forum, pp 43-49, 1997.

[3] M. Sindou, G. Ablart, C. Sournois, Multiband and wideband properties of printed fractal branched antennas, Electro. lett, 35(3):181-2, February 1999.

[4] Puente, Claret, J, Sagues, F, Romeu. J, Lopez-Salvans, M.Q, and R. Pous, Multiband properties of a fractal Tree antenna generated by electrochemical deposition, Electron. Lett, 1996, pp 2298-2299. 
[5] J. S. Petko, D. Werner, Miniature reconfigurable three dimensional fractal tree antenna, IEEE Trans. Antennas and Propagation. August 2004.

[6] P. Simedrea, Design and implementation of compact microstrip fractal antennas, University of Western Ontario, London, Thesis, March 2004.

[7] C. A. Balanis, Antenna theory, analysis and design, third edition, Wiley \& Sons, 2005.

[8] J. Gianvittorio, Y. Rahmat-Samii, Fractal element antennas: a compilation of configurations with novel characteristics, IEEE AP-S International Symposium, Salt Lake City, 2000.

[9] R. Breden, R. J. Langley, Printed fractal antennas, Proc. Inst. Elect. Eng. National Conf. Antennas and Propagation, vol. 461, pp. 1-4, 1999.

[10] J. Gianvittorio, Fractal antennas design, characterisation, and applications, Master Thesis of science in electrical engineering, university of California, Los Angeles, 2000.

\section{Nomenclatures:}

$s \quad$ scale factor

$\theta \quad$ The branching half angle.

$\varepsilon_{\text {eff }}$ effective dielectric constant of substrate

$h$ height of antenna

$\lambda_{0} \quad$ wavelength in free space

$\varepsilon_{0} \quad$ dielectric constant of substrate

$w \quad$ width of a strip

$h_{0} \quad$ height of the substrate 